OPEN ACCESS

Edited by:

Steffen Tiedt,

LMU Munich University

Hospital, Germany

Reviewed by:

Christoph Riegler,

Charité - Universitätsmedizin

Berlin, Germany

Anna Christina Alegiani,

University Medical Center

Hamburg-Eppendorf, Germany

Wei Zhao,

Shandong University, China

${ }^{*}$ Correspondence:

Jian Xia

xjian1216@csu.edu.cn

tThese authors have contributed equally to this work and share first

authorship

Specialty section:

This article was submitted to

Stroke,

a section of the journa

Frontiers in Neurology

Received: 24 May 2021 Accepted: 03 August 2021 Published: 26 August 2021

Citation:

Yu F, Zhang L, Liao D, Luo Y, Feng $X$, Liu Z and Xia J (2021) Serum Bilirubin

Levels and Extent of Symptomatic Intracranial Atherosclerotic Stenosis in Acute Ischemic Stroke: A

Cross-Sectional Study.

Front. Neurol. 12:714098 doi: 10.3389/fneur.2021.714098

\section{Serum Bilirubin Levels and Extent of Symptomatic Intracranial Atherosclerotic Stenosis in Acute Ischemic Stroke: A Cross-Sectional Study}

\author{
Fang Yu ${ }^{1+}$, Lin Zhang ${ }^{1 \dagger}$, Di Liao ${ }^{1}$, Yunfang Luo ${ }^{1}$, Xianjing Feng ${ }^{1}$, Zeyu Liu ${ }^{1}$ and Jian Xia ${ }^{1,2,3 *}$ \\ ${ }^{1}$ Department of Neurology, Xiangya Hospital, Central South University, Changsha, China, ${ }^{2}$ Clinical Research Center for \\ Cerebrovascular Disease of Hunan Province, Central South University, Changsha, China, ${ }^{3}$ National Clinical Research Center \\ for Geriatric Disorders, Xiangya Hospital, Central South University, Changsha, China
}

Background: Bilirubin plays a paradoxical role in the pathological mechanism of stroke. To date, few clinical studies have investigated the effect of serum bilirubin on symptomatic intracranial atherosclerotic stenosis (sICAS). This study aims to evaluate the connection between serum bilirubin and sICAS.

Methods: From September 2015 to May 2020, 1,156 sICAS patients without hepatobiliary diseases admitted to our hospital were included. Patients were distributed into none-mild (0-49\%), moderate (50-69\%) and severe-occlusion sICAS groups (70-100\%) by the degree of artery stenosis. Moderate and severe-occlusion sICAS patients were classified into three groups by the number of stenotic arteries (single-, two- and multiple-vessel stenosis). The relationship between serum bilirubin levels and sICAS was analyzed by logistic regression analysis.

Results: In univariable analyses, sICAS patients with severe and multiple atherosclerotic stenoses had lower levels of total bilirubin (Tbil), direct bilirubin (Dbil), and indirect bilirubin (Ibil). In multinomial logistic regression analyses, when compared with the highest tertile of bilirubin, lower levels of Tbil, Dbil, and Ibil showed higher risks of severe-occlusion sICAS (95\% Cl: 2.018-6.075 in tertile 1 for Tbil; 2.380-7.410 in tertile 1 for Dbil; 1.758-5.641 in tertile 1 for lbil). Moreover, the logistic regression analyses showed that lower levels of Tbil, Dbil, and Ibil were related to multiple ( $\geq 3$ ) atherosclerotic stenoses (95\% Cl: 2.365-5.298 in tertile 1 and 2.312-5.208 in tertile 2 for Tbil; 1.743-3.835 in tertile 1 and 1.416-3.144 in tertile 2 for Dbil; $2.361-5.345$ in tertile 1 and 1.604-3.545 in tertile 2 for (bil) when compared with tertile 3.

Conclusions: Our findings suggest that lower bilirubin levels may indicate severe and multiple intracranial atherosclerotic stenoses.

Keywords: ischemic stroke, symptomatic intracranial atherosclerotic stenosis, bilirubin, biomarkers, oxidative stress 


\section{INTRODUCTION}

Symptomatic intracranial atherosclerosis (sICAS), a critical cause of ischemic stroke in China, refers to the stenosis $\geq 50 \%$ of one or more intracranial arteries (1). In Asia, $\sim 50 \%$ of patients with transient ischemic attack (TIA) and $40 \%$ of patients with ischemic stroke (IS) have ICAS $(2,3)$. sICAS is a developing and dynamically changing disease with a high recurrence risk, causing a huge social burden. Traditional risk factors such as age, ethnicity, obesity, hypertension, diabetes, hyperlipidemia, smoking, and metabolic syndrome have been reported to be closely related to sICAS (4). However, the relationship between circulating biomarkers and sICAS is less explored.

Bilirubin is produced by heme catabolism, including total bilirubin (Tbli), indirect bilirubin (Ibil), and direct bilirubin (Dbil). In the past few decades, bilirubin has been considered as a potentially toxic metabolite, which could damage the central nervous system once passing through the blood-brain barrier $(5,6)$. However, subsequent evidence has shown that bilirubin plays a dual role in oxidative stress and it may be a protective factor for atherosclerosis $(7,8)$. Accumulating research suggests that bilirubin can inhibit the production of oxidized lowdensity lipoprotein (ox-LDL), increase the solubility of serum cholesterol, inhibit protein kinase $\mathrm{C}$ activity in human fibroblasts, and capture oxygen free radicals, thus inhibiting the progression of atherosclerosis (9-11).

Several clinical observations have indicated that high bilirubin concentrations could reduce the risk of stroke (12-14). Besides, a small number of studies have revealed the negative associations between high bilirubin levels and the occurrence of asymptomatic intracranial atherosclerosis (aICAS) (15) and extracranial atherosclerosis (16). However, research to date has not yet determined the relationship between bilirubin and sICAS.

Therefore, this research aimed to explore the relationship between serum bilirubin concentrations (including Tbil, Dbil, and Ibil) and sICAS in the Chinese Han Population.

\section{METHODS}

\section{Study Population}

This study was a descriptive, retrospective, cross-sectional study. From September 2015 to May 2020, patients with TIA or acute ischemic stroke (AIS) caused by large artery atherosclerosis (LAA) within 14 days from symptom onset were enrolled from the Department of Neurology of Xiangya Hospital. The information of all patients was collected from the medical records. The diagnosis of AIS and TIA matched with the 2018 Chinese AIS guidelines (17). We assessed the stroke severity of AIS patients at admission using the National Institutes of Health Stroke Scale (NIHSS) score. All patients were subtyped by the Chinese ischemic stroke subclassification (CISS) system (18). We recruited 1,015 patients caused by intracranial atherosclerotic stenosis (stenosis $\geq 50 \%$ ) and 141 patients attributed to atherosclerotic causes with none-mild intracranial stenosis (stenosis: 0-49\%). Exclusion criteria were as follows: (1) under 18 years old; (2) other causes for TIA or IS such as small vessel occlusion or cardioembolism;
(3) incomplete clinical information or laboratory tests; (4) patients with extracranial artery stenosis diagnosed by carotid contrast-enhanced magnetic resonance angiography (CE-MRA) or carotid computed tomography angiography (CTA) according to the methods used in the North American Symptomatic Carotid Endarterectomy Trial (19); (5) brain tumor, intracranial or systemic infection, congenital hypoplastic cerebrovascular disease, etc; (6) other diseases causing intracranial artery stenosis such as vascular malformation, moyamoya disease, artery dissection, vasculitis and syphilis (20); (7) suffering from hepatobiliary diseases (Tbil $>34.2 \mu \mathrm{mol} / \mathrm{L}$, alanine aminotransferase $[\mathrm{ALT}] \geq 80 \mathrm{IU} / \mathrm{L}$, aspartate aminotransferase $\geq 80 \mathrm{IU} / \mathrm{L}$, serum albumin $<3.5 \mathrm{~g} / \mathrm{dL}$ ) or other diseases that may affect bilirubin level such as Gilbert syndrome (Tbil $>34.2$ $\mu \mathrm{mol} / \mathrm{L}, \mathrm{ALT}<80 \mathrm{IU} / \mathrm{L}$, aspartate aminotransferase $<80 \mathrm{IU} / \mathrm{L}, \gamma-$ glutamyl transpeptidase $<80 \mathrm{IU} / \mathrm{L})(21)$. This study was approved by the Ethics Committees of Xiangya Hospital of Central South University, Changsha, Hunan Province, China (ethical approval number: 201503330). All patients or their family members signed the informed consent.

\section{Demographics and Risk Factors}

The following clinical information was collected via questionnaires and physical examinations: age, sex, hypertension, diabetes mellitus, hyperlipemia, alcohol use, smoking duration, and the history of coronary artery disease. Hypertension was defined as systolic blood pressure $\geq 140 \mathrm{mmHg}$ or diastolic blood pressure $\geq 90 \mathrm{mmHg}$ or currently taking antihypertensive drugs (22). Diagnostic criteria for diabetes: random blood glucose $\geq 11.1 \mathrm{mmol} / \mathrm{L}$, fasting blood glucose $\geq 7.0 \mathrm{mmol} / \mathrm{L}$ or using hypoglycemic drugs (23). Dyslipidemia was diagnosed as serum triglyceride $\geq 1.7 \mathrm{mmol} / \mathrm{L}$, serum total cholesterol $\geq 5.2 \mathrm{mmol} / \mathrm{L}$, serum low-density lipoprotein cholesterol $\geq 3.4 \mathrm{mmol} / \mathrm{L}$, or serum high-density lipoprotein $<1.0 \mathrm{mmol} / \mathrm{L}$ or using antihyperlipidemic drugs (24). Smoking was determined based on the self-report questionnaire at the time of admission, and the smoking amount was defined as pack-years in our study (25). The pack-years was measured based on the average smoking volume and the past and current smoking durations (25). All patients were divided into four groups according to smoking mount: group 1 (0, non-smoker), group 2 (0-15 pack-years), group 3 (15-30 pack-years), and group 4 (>30 pack-years) (25). The state of alcoholism was thought to be an average of more than $20 \mathrm{~g}$ of alcohol per day (26). Fasting overnight, the blood samples of all patients were collected the next morning after admission (within 14 days of stroke onset) and sent to the same laboratory department in our hospital. The data of white blood cell, Tbil, Dbil, Ibil, total cholesterol, triglyceride, high-density lipoprotein, low-density lipoprotein, fasting blood glucose, glycosylated hemoglobin A1c, uric acid, and homocysteine levels were derived from the medical records.

\section{Radiological Assessment}

On admission, magnetic resonance imaging (MRI) and time of flight magnetic resonance angiography (TOF-MRA) were performed for most patients. Computed tomography (CT) and computed tomography angiography (CTA) were performed for 
patients with contraindications to MRI. In addition, CTA was performed when there were doubts about the results of MRA, and digital subtraction angiography (DSA) was performed when the results of MRA and CTA were inconsistent. Meanwhile, carotid CTA and carotid CE-MRA were used to exclude extracranial artery stenosis. All imaging data were evaluated by at least two neurologists with more than 5 years of experience. They knew nothing about the clinical information and reached a consensus. According to the results of MRA, CTA, DSA, or CE-MRA, ICAS was diagnosed as large intracranial artery stenosis (50$100 \%$ ), including bilateral internal carotid artery (ICA), bilateral anterior cerebral artery (ACA), bilateral middle cerebral artery (MCA), bilateral posterior cerebral artery (PCA), bilateral basilar artery (BA) or bilateral vertebral artery (VA) (27). The degree of intracranial stenosis was assessed by MRA/CTA/DSA using Warfarin-Aspirin Symptomatic Intracranial Disease (WASID) method with reference to normal distal vessels (28). Patients with none-mild intracranial stenosis (stenosis: 0-49\%) served as controls. In this study, participants were divided into none-mild group (0-49\%), moderate group (50-69\%), and severe-occlusion group (70-100\%) based on the degree of artery stenosis. We then counted the number of intracranial stenotic arteries (stenosis $\geq 50 \%$ ) of ICAS patients and classified patients into three groups (single-, two- and multiple-vessel stenosis) accordingly.

\section{Statistical Analysis}

The statistical analysis was conducted using IBM SPSS Statistics 22.0 (Chicago, USA), and all data were expressed as frequency (\%) and the median (interquartile range, IQR). Characteristics of the objects were compared with a chi-square $\left(\chi^{2}\right)$ test for categorical variables and the Mann-Whitney $U$ test or KruskalWallis test for continuous variables. We also analyzed the association between bilirubin and sICAS in different groups according to the degree and number of vascular stenosis. Bilirubin levels were categorized into tertiles and the $\chi^{2}$ test for trends was used to analyze the dose-effect of Tbil, Dbil, and Ibil. Factors with $P<0.05$ in univariate analysis and reported confounding risk factors were included in multivariate logistic regression analysis to evaluate the independent influence of bilirubin. Tbli, Dbil, and Ibil were tested separately to avoid interaction. We used multinomial logistic regression instead of ordinal polytomous logistic regression because the test of the parallel lines hypothesis was rejected. The results were shown by odds ratio (OR) and 95\% confidence interval (CI). In addition, receiver operating characteristic curve (ROC) analysis was conducted by MedCalc software (MedCalc Inc., Mariakerke, Belgium) to determine the predictability of bilirubin for discriminating the extent of ICAS. $P<0.05$ was considered significant.

\section{RESULTS}

\section{Clinical Characteristics of Patients With Ischemic Stroke}

Clinical characteristics of all patients were presented in Table $\mathbf{1 .}$ A total of 1,156 subjects were finally included in the study. The average age of the participants was 61 (IQR, 53-68) years old and
TABLE 1 | Baseline characteristics of patients with ischemic stroke.

\begin{tabular}{|c|c|}
\hline Characteristics & Value \\
\hline Age years [IQR] & $61[53-68]$ \\
\hline Sex (male, $N, \%$ ) & $757(65.5)$ \\
\hline Hypertension (N, \%) & $872(75.4)$ \\
\hline Diabetes mellitus ( $N, \%)$ & $389(33.7)$ \\
\hline Hyperlipidemia (N, \%) & $521(45.1)$ \\
\hline $\operatorname{CAD}(N, \%)$ & $178(15.4)$ \\
\hline \multicolumn{2}{|c|}{ Smoking duration (pack-years) $(N, \%)$} \\
\hline Group $1(0)$ & $638(55.2)$ \\
\hline Group $2(>0, \leq 15)$ & $107(9.3)$ \\
\hline Group $3(>15, \leq 30)$ & $184(15.9)$ \\
\hline Group 4 (>30) & $227(19.6)$ \\
\hline Drinking (N, \%) & $376(32.5)$ \\
\hline NIHSS [IQR] & $4[2-8]$ \\
\hline SBP mmHg [IQR] & 144 [132-158] \\
\hline DBP mmHg [IQR] & 84 [76-93] \\
\hline WBC,$\times 10^{9} / \mathrm{L}[\mathrm{IQR}]$ & $6.80[5.60-8.40]$ \\
\hline Tbil, $\mu \mathrm{mol} / L$ [IQR] & 10.34 [7.70-13.40] \\
\hline Dbil, $\mu \mathrm{mol} / L$ [IQR] & 4.50 [3.30-5.90] \\
\hline Ibil, $\mu \mathrm{mol} / L$ [IQR] & 5.60 [4.00-7.90] \\
\hline FBG, mmol/L [IQR] & $5.60[4.91-7.18]$ \\
\hline $\mathrm{HbA1c}, \%[\mathrm{IQR}]$ & $5.80[5.70-6.60]$ \\
\hline $\mathrm{TC}, \mathrm{mmol} / \mathrm{L}[\mathrm{IQR}]$ & $4.37[3.55-5.18]$ \\
\hline $\mathrm{TG}, \mathrm{mmol} / \mathrm{L}[\mathrm{IQR}]$ & $1.55[1.16-2.12]$ \\
\hline $\mathrm{HDL}, \mathrm{mmol} / \mathrm{L}[\mathrm{IQR}]$ & $1.02[0.87-1.22]$ \\
\hline LDL, mmol/L [IQR] & 2.66 [2.05-3.30] \\
\hline UA, $\mu \mathrm{mol} / \mathrm{L}[\mathrm{IQR}]$ & 316.20 [256.20-382.15] \\
\hline $\mathrm{HCY}, \mu \mathrm{mol} / \mathrm{L}[\mathrm{IQR}]$ & 13.25 [11.11-16.35] \\
\hline
\end{tabular}

IQR, Inter quartile range; CAD, coronary artery disease; NIHSS, National Institute of Health stroke scale; SBP, systolic blood pressure; DBP, diastolic blood pressure; WBC, white blood cell; Tbil, total bilirubin; Dbil, direct bilirubin; Ibil, indirect bilirubin; FBG, fasting blood glucose; HbA1c, glycosylated hemoglobin A1C; TC, total cholesterol; TG, triglyceride; HDL, high density lipoprotein; $L D L$, low density lipoprotein; UA, Uric acid; HCY, Homocysteine. Smoking duration: group 1 (0, non-smoker), group 2 (0-15 pack-years), group 3 (15-30 pack-years) and group 4 (>30 pack-years).

$65.5 \%$ were male. The median (IQR) of Tbil, Dbil, and Ibli levels was 10.34 (7.70-13.40), $4.50(3.30-5.90)$, and $5.60(4.91-7.18)$ $\mu \mathrm{mol} / \mathrm{L}$, separately.

\section{Baseline Characteristics of the Study Population According to the Severity of ICAS}

According to the degree of intracranial artery stenosis, participants were divided into none-mild group $(0-49 \%, n=$ $141)$, moderate group $(50-69 \%, n=357)$, and severe-occlusion group (70-100\%, $n=658)$. Baseline clinical characteristics and laboratory tests of these 1,156 objects were shown in Table 2. There were significant differences among these three groups in sex, hypertension, hyperlipidemia, systolic blood pressure, diastolic blood pressure, and the levels of Tbil, Dbil, Ibil, uric acid, and homocysteine. Moreover, Figures 1A-C illustrates the median concentrations of Tbil, Dbil and Ibil in different 
TABLE 2 | Baseline characteristics of the study population according to the severity of ICAS.

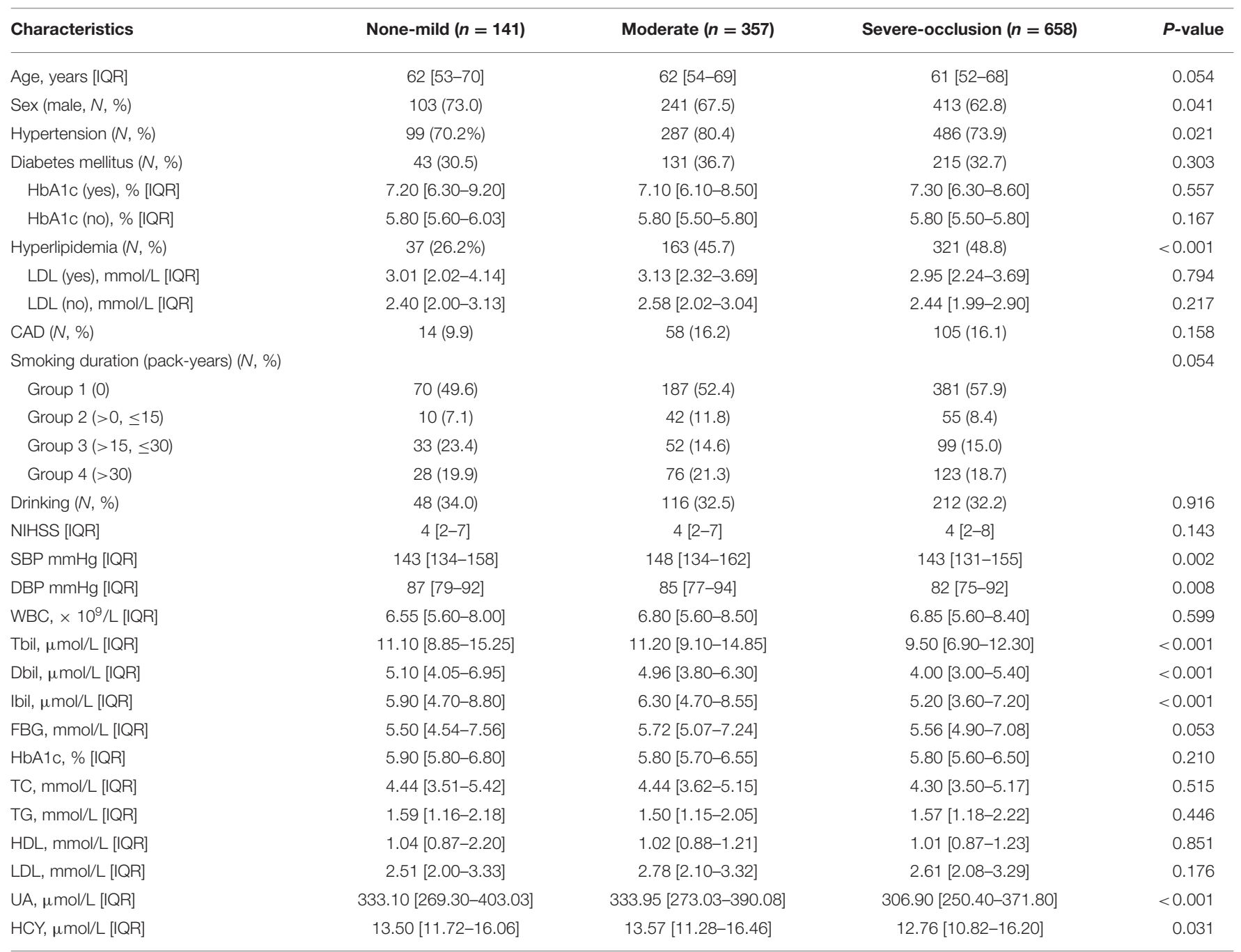

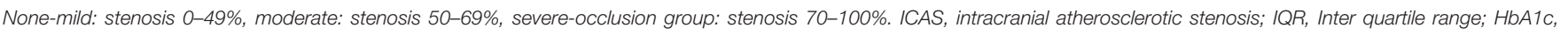

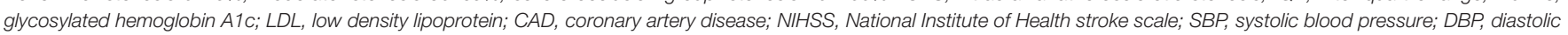

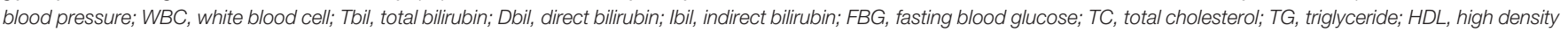

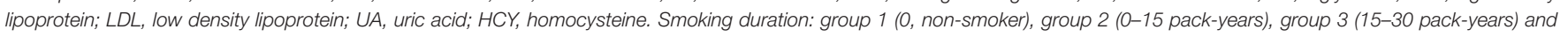
group 4 (>30 pack-years).

groups according to the severity of ICAS. Lower bilirubin levels were found in the severe-occlusion group compared with the none-mild or moderate group. Bilirubin levels were categorized into tertiles, and the linear trends across the three categories were tested by chi-square linear trend test. The chi-square linear trend test yielded a significant result $(P$-trends $<0.001)$ (Figure 1D), mostly due to the significant difference in bilirubin levels between the moderate and severe-occlusion ICAS groups. The serum bilirubin levels in patients with none-mild ICAS were similar compared with patients presenting with moderate ICAS. We tried to find clear cutoff values by ROC analyses to discriminate the severity of ICAS, however, the performance of these models was rather poor with AUCs of 0.627-0.640. The results were shown in Supplementary Figure 1A and Supplementary Table 1.

\section{Baseline Characteristics of the Study Population Based on the Number of Stenotic Arteries}

Based on the number of stenotic arteries, patients were stratified into three groups: single-vessel stenosis $(n=294)$, two-vessel stenosis $(n=193)$ and multiple-vessel stenosis $(n=528)$. Table 3 provides the specific clinical information and laboratory results. There were significant differences among the three groups in age, hypertension, diabetes, hyperlipidemia, Tbil, Dbil, Ibil, fasting blood glucose, and glycosylated hemoglobin Alc. In addition, lower bilirubin levels were found in the multiple-vessel stenosis group compared with the single- and two-vessel stenosis groups (as shown in Figures 1E-G). The chi-square linear trend test yielded a significant result $(P$-trends $<0.001$ ) (Figure $\mathbf{1 H}$ ), mostly due to the significant difference in bilirubin levels between the 


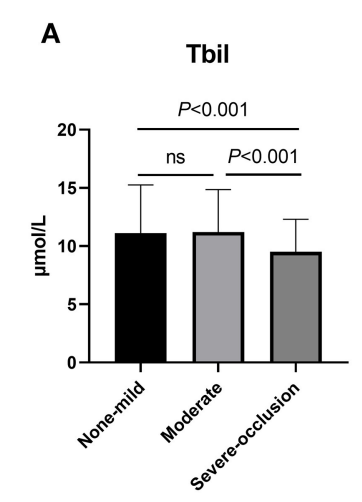

B
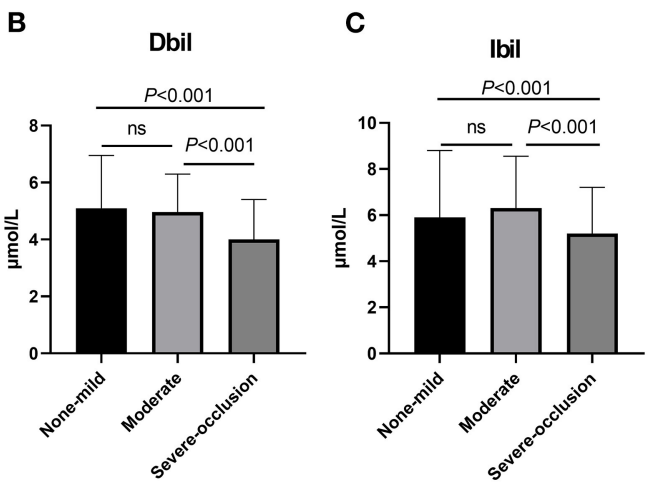

D
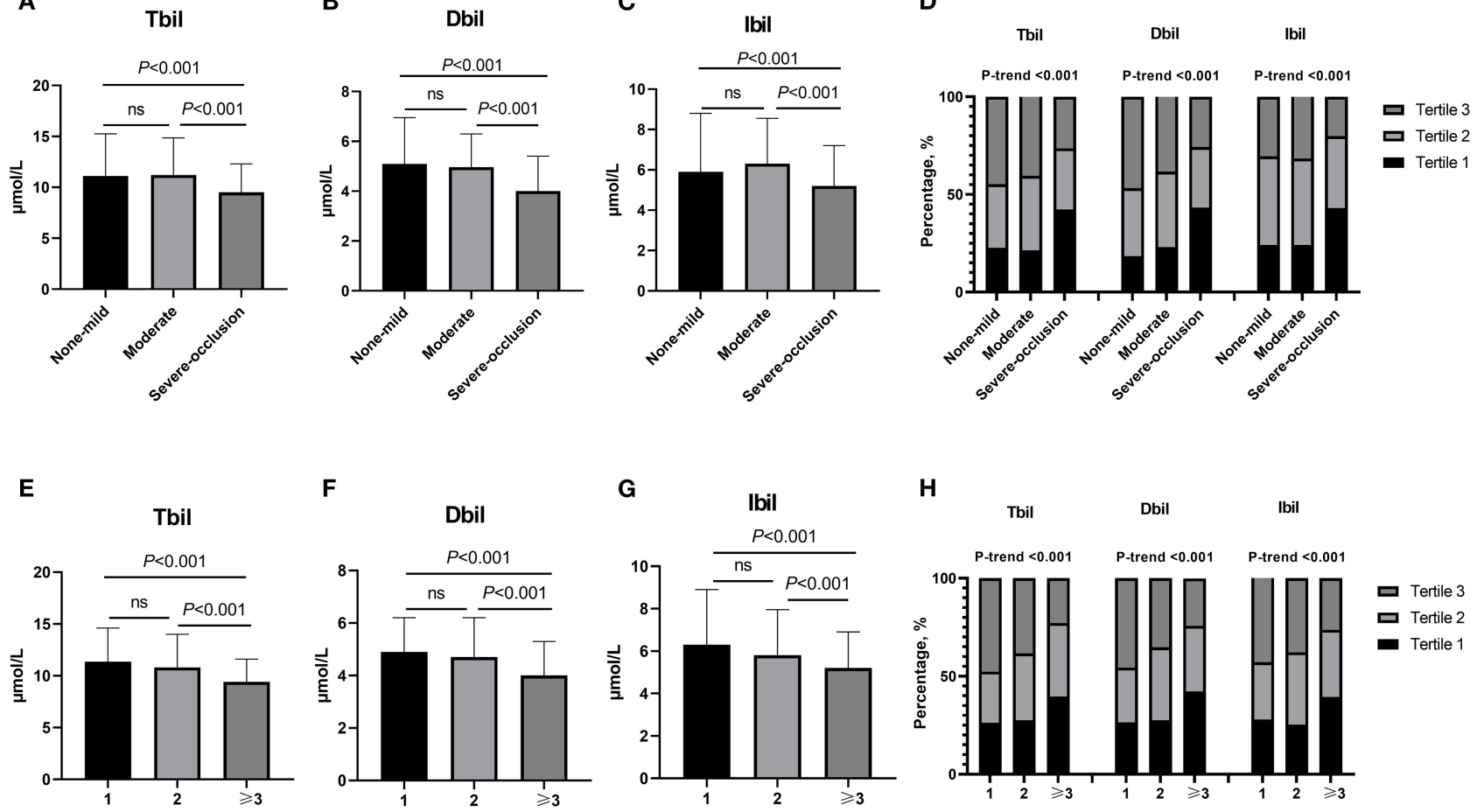

$\mathbf{F}$

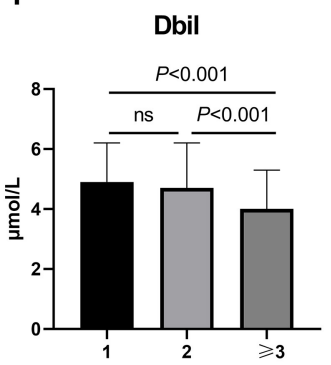

G

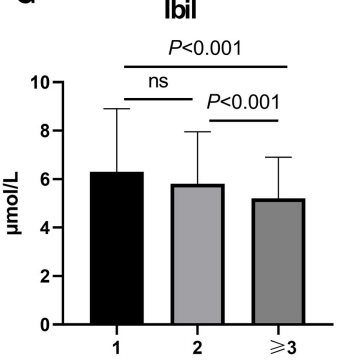

H

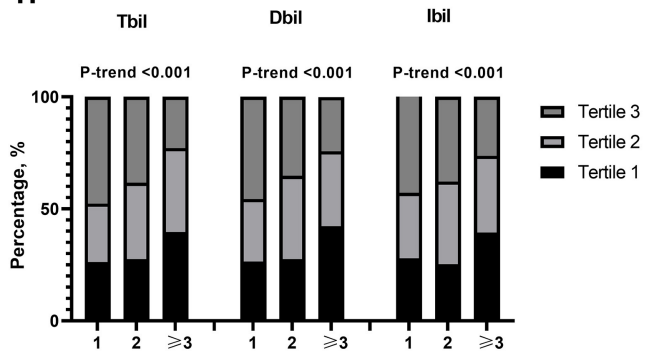

FIGURE 1 | (A-D) Bilirubin levels in different groups stratified by the severity of ICAS (none-mild group: stenosis 0-49\%, moderate group: stenosis 50-69\% and severe-occlusion group: stenosis 70-100\%). (A) Different Tbli levels among non-mild, moderate and severe-occlusion groups. Tertile 1: $<8.6 \mu \mathrm{mol} / \mathrm{L}$, Tertile 2: 8.6-12.1 $\mu \mathrm{mol} / \mathrm{L}$, Tertile 3: $>12.1 \mu \mathrm{mol} / \mathrm{L}$. (B) Different Dbli levels among non-mild, moderate and severe-occlusion groups. Tertile 1: $<3.7 \mu \mathrm{mol} / \mathrm{L}$, Tertile 2: 3.7-5.3 $\mu \mathrm{mol} / \mathrm{L}$, Tertile 3: $>5.3 \mu \mathrm{mol} / \mathrm{L}$. (C) Different Ibli levels among non-mild, moderate and severe-occlusion groups. Tertile 1: $<4.6 \mu \mathrm{mol} / \mathrm{L}$, Tertile $2: 4.6-7.9 \mu \mathrm{mol} / \mathrm{L}$, Tertile 3: $>7.9 \mu \mathrm{mol} / \mathrm{L}$. (D) The different percentages of Tbli, Dbli and Ibli tertiles among non-mild, moderate and severe-occlusion groups. (E-H) Different bilirubin levels in groups according to the number of stenoses $(\geq 50 \%)(n=1,2$, and $\geq 3)$. (E) Different Tbli levels among groups. Tertile 1: $<8.4 \mu \mathrm{mol} / \mathrm{L}$, Tertile 2: 8.4-11.9 $\mu \mathrm{mol} / \mathrm{L}$, Tertile 3: $>11.9 \mu \mathrm{mol} / \mathrm{L}$. (F) Different Dbli levels among groups. Tertile 1: $<3.6 \mu \mathrm{mol} / \mathrm{L}$, Tertile 2: 3.6-5.1 $\mu \mathrm{mol} / \mathrm{L}$, Tertile 3: $>5.1 \mu \mathrm{mo} / \mathrm{L}$. (G) Different Ibli levels among groups. Tertile 1: $<4.5 \mu \mathrm{mol} / \mathrm{L}$, Tertile 2: $4.5-6.9 \mu \mathrm{mol} / \mathrm{L}$, Tertile 3: $>6.9 \mu \mathrm{mol} / \mathrm{L}$. (H) The different percentages of Tbli, Dbli and Ibli tertiles in groups according to the number of stenoses $(\geq 50 \%)(n=1,2$, and $\geq 3) . n=1$, single-vessel stenosis; $n=2$, two-vessel stenosis; $n \geq 3$, multiple-vessel stenosis. ICAS, intracranial atherosclerotic stenosis; Tbil, total bilirubin; Dbil, direct bilirubin; Ibil, indirect bilirubin.

two- and multiple-vessel stenosis groups. ROC analyses were used to differentiate the number of stenotic arteries in patients with ICAS, unfortunately, the ROC methods demonstrated poor discriminatory capability for constructing cutoff values (AUCs: $0.600,0.607$, and 0.615). The results were presented in the Supplementary Figure 1B and Supplementary Table 1.

\section{Multivariate Logistic Regression Analyses for Predictors of Severe ICAS}

The findings obtained from the multivariate logistic regression analysis are presented in Table 4. The variables with $P<0.05$ in univariate analysis in Table 2 and other reported confounding factors were input into the multivariate model. Tbil, Dbil, and Ibil did not have significant influence on the degree of ICAS when comparing the group with moderate ICAS with the nonemild ICAS group. When comparing the severe-occlusion and none-mild groups, lower Tbil, Dbil, and Ibil levels were found to be independent factors (OR: 3.502, 95\% CI: $2.018-6.075, P$ $<0.001$ in tertile 1 for Tbil; OR: $4.199,95 \%$ CI: $2.380-7.410, P$ $<0.001$ in tertile 1 for Dbil; OR: $3.149,95 \%$ CI: $1.758-5.641$,
$P<0.001$ in tertile 1 for Ibil) compared to the highest levels of bilirubin.

\section{Multivariate Logistic Regression Analyses for Predictors of Multi-Stenosis of ICAS}

Table 5 shows the results of multinomial logistic regression analysis. We compared two- and multiple-vessel stenosis groups separately, with single-vessel stenosis group as the control. Using tertile 3 as a reference, we found that lower levels of Tbil, Dbil, and Ibil were related to multiple (two-vessel stenosis or multiplevessel stenosis) atherosclerotic stenosis (when two- vs. singlevessel stenosis, OR: 2.052, 95\% CI: $1.270-3.314, P=0.003$ in tertile 2 for Tbil; OR: 1.866, 95\% CI: 1.167-2.986, $P=0.009$ in tertile 2 for Ibil. When multiple- vs. single-vessel stenosis, OR: 3.540, 95\% CI: $2.365-5.298, P<0.001$ in tertile 1 and OR: 3.470 , 95\% CI: 2.312-5.208, $P<0.001$ in tertile 2 for Tbil; OR: 2.585, 95\% CI: $1.734-3.835, P<0.001$ in tertile 1 and OR: $2.110,95 \%$ CI: $1.416-3.144, P<0.001$ in tertile 2 for Dbil; OR: 3.552, 95\% CI: $2.361-5.345, P<0.001$ in tertile 1 and OR: $2.384,95 \% \mathrm{CI}$ : 1.604-3.545, $P<0.001$ in tertile 2 for Ibil). 
TABLE 3 | Baseline characteristics of the participants according to the number of stenotic arteries.

\begin{tabular}{|c|c|c|c|c|}
\hline Characteristics & $1(n=294)$ & $2(n=193)$ & $\geq 3(n=528)$ & $P$-value \\
\hline Age years [IQR] & $57[48-66]$ & 62 [53-69] & 63 [54-69] & $<0.001$ \\
\hline Sex (male, $N, \%)$ & $204(69.4)$ & $127(65.8)$ & $323(661.2)$ & 0.056 \\
\hline Hypertension (N, \%) & $191(65.0)$ & $154(79.8)$ & $428(81.1)$ & $<0.001$ \\
\hline Diabetes mellitus ( $N, \%)$ & $78(26.5)$ & $69(35.8)$ & $199(37.7)$ & 0.005 \\
\hline HbA1c (yes), \% [IQR] & 6.65 [5.80-7.80] & $7.20[6.20-8.70]$ & $7.40[6.40-8.60]$ & 0.002 \\
\hline LDL (yes), mmol/L [IQR] & 3.06 [2.25-3.70] & 3.06 [2.20-3.55] & 2.95 [2.28-3.72] & 0.896 \\
\hline LDL (no), mmol/L [IQR] & 2.45 [1.94-2.94] & 2.56 [2.11-2.94] & $2.47[2.02-2.97]$ & 0.400 \\
\hline CAD (N, \%) & $42(14.3)$ & $34(17.6)$ & $88(16.7)$ & 0.559 \\
\hline Smoking duration (pack-years) $(\mathrm{N}, \%)$ & & & & 0.092 \\
\hline Group 1 (0) & $152(51.7)$ & $105(54.4)$ & $311(58.9)$ & \\
\hline Drinking $(N, \%)$ & $94(32.0)$ & $71(36.8)$ & $163(30.9)$ & 0.319 \\
\hline NIHSS [IQR] & $5[2-8]$ & $5[2-8]$ & $4[2-7]$ & 0.151 \\
\hline $\mathrm{SBP} \mathrm{mmHg}[\mathrm{IQR}]$ & 144 [128-155] & $143[134-158]$ & 145 [133-159] & 0.078 \\
\hline DBP mmHg [IQR] & 84 [76-95] & 85 [77-93] & 82 [75-91] & 0.128 \\
\hline WBC, $\times 10^{9} / \mathrm{L}[\mathrm{IQR}]$ & $6.90[5.60-8.50]$ & 7.20 [5.60-8.78] & $6.70[5.60-8.20]$ & 0.220 \\
\hline Tbil, $\mu \mathrm{mol} / \mathrm{L}$ [IQR] & $11.35[8.10-14.60]$ & $10.80[8.30-14.00]$ & $9.40[7.03-11.60]$ & $<0.001$ \\
\hline Dbil, $\mu \mathrm{mol} / \mathrm{L}[\mathrm{IQR}]$ & 4.90 [3.58-6.20] & $4.70[3.60-6.20]$ & $4.00[3.00-5.30]$ & $<0.001$ \\
\hline Ibil, $\mu \mathrm{mol} / \mathrm{L}[\mathrm{IQR}]$ & 6.30 [4.40-8.90] & 5.80 [4.45-7.95] & 5.20 [3.70-6.90] & $<0.001$ \\
\hline FBG, mmol/L [IQR] & 5.50 [4.91-6.69] & 5.92 [5.09-7.53] & 5.67 [4.93-7.22] & 0.032 \\
\hline $\mathrm{HbA1c}, \%[\mathrm{IQR}]$ & 5.80 [5.60-6.10] & 5.80 [5.70-6.95] & 5.80 [5.70-6.78] & $<0.001$ \\
\hline TC, mmol/L [IQR] & $4.38[3.50-5.30]$ & $4.41[3.65-5.14]$ & $4.34[3.57-5.11]$ & 0.498 \\
\hline
\end{tabular}

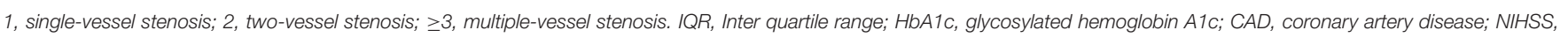

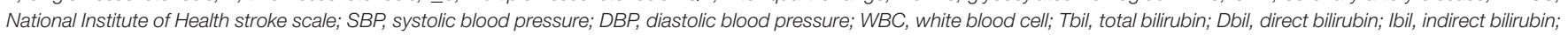

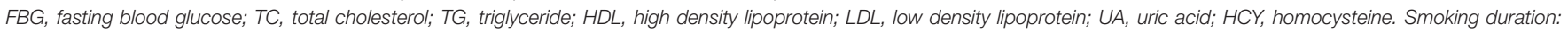
group 1 (0, non-smoker), group 2 (0-15 pack-years), group 3 (15-30 pack-years) and group 4 (>30 pack-years).

\section{DISCUSSION}

The present study indicates that patients with severe and multiple sICAS had significantly lower serum bilirubin levels, even after adjusting for confounding factors such as age, sex, smoking status, hypertension, diabetes and hyperlipidemia.

ICAS is a leading cause of stroke occurrence and recurrence worldwide and is associated with higher risk for ischemic stroke and death. Since atherosclerosis is a chronic disease mediated by endothelial dysfunction, lipid deposition and inflammation, oxidative stress might play a crucial role in the pathological processes of sICAS (29-32). Prior studies have shown that endothelial dysfunction acts in the preclinical development of atherosclerosis, and inflammation could increase the vulnerability of plaques. Under oxidative stress, LDL could be transformed into oxidized low-density lipoprotein (ox-LDL), and vascular endothelial dysfunction and increased permeability could promote the deposition of ox-LDL in the intima (33). Furthermore, the accumulated ox-LDL contributes to the initiation of inflammatory reactions, infiltration of monocytes and $\mathrm{T}$ cells, and accumulation of extracellular matrix (34). $\mathrm{T}$ cells could recognize antigens and initiate the Type-1 immunity, causing local inflammation and plaque growth, leading to gradual narrowing of blood vessels and ICAS development (34).

Bilirubin has antioxidant and anti-inflammatory activities, reported to be inversely correlated with asymptomatic intracranial atherosclerosis $(15,35,36)$. The mechanisms by which bilirubin functions in ICAS remains unclear, but prior studies indicate that bilirubin could inhibit atherosclerosis in several ways. First, reactive oxygen species promote lipid peroxidation, endothelial cell injury, smooth muscle cell proliferation and migration, inflammatory factor expression, and 
TABLE 4 | Multivariate logistic regression analyses for predictors of severe-occlusion of ICAS.

\begin{tabular}{|c|c|c|c|c|c|c|c|c|c|c|c|c|}
\hline & \multicolumn{2}{|c|}{ Moderate vs. None-mild } & \multicolumn{2}{|c|}{$\begin{array}{l}\text { Severe-occlusion vs. } \\
\text { None-mild }\end{array}$} & \multicolumn{2}{|c|}{ Moderate vs. None-mild } & \multicolumn{2}{|c|}{$\begin{array}{l}\text { Severe-occlusion vs. } \\
\text { None-mild }\end{array}$} & \multicolumn{2}{|c|}{ Moderate vs. None-mild } & \multicolumn{2}{|c|}{$\begin{array}{l}\text { Severe-occlusion vs. } \\
\text { None-mild }\end{array}$} \\
\hline & $P$-value & OR (95\% Cl) & $P$-value & OR (95\% Cl) & $P$-value & OR (95\% Cl) & $P$-value & OR (95\% Cl) & $P$-value & OR (95\% Cl) & $P$-value & OR (95\% Cl) \\
\hline Age, years & 0.768 & 0.997 (0.975-1.019) & 0.116 & $0.984(0.964-1.004)$ & 0.803 & $0.997(0.976-1.019)$ & 0.118 & $0.984(0.964-1.004)$ & 0.775 & 0.997 (0.975-1.019) & 0.129 & $0.984(0.964-1.005)$ \\
\hline Sex, (Female vs. male) & 0.416 & $0.771(0.412-1.444)$ & 0.926 & $1.029(0.569-1.860)$ & 0.538 & $0.820(0.437-1.541)$ & 0.908 & $1.036(0.571-1.878)$ & 0.433 & $0.778(0.415-1.457)$ & 0.967 & $1.013(0.561-1.829)$ \\
\hline Hypertension & 0.045 & $1.773(1.013-3.104)$ & 0.236 & $1.364(0.816-2.280)$ & 0.062 & $1.700(0.973-2.972)$ & 0.238 & $1.362(0.815-2.275)$ & 0.042 & $1.788(1.022-3.127)$ & 0.206 & $1.392(0.834-2.322)$ \\
\hline Diabetes mellitus & 0.376 & $1.245(0.767-2.020)$ & 0.999 & $1.000(0.629-1.590)$ & 0.450 & $1.206(0.743-1.957)$ & 0.875 & $0.963(0.605-1.534)$ & 0.356 & $1.255(0.774-2.034)$ & 0.884 & $1.035(0.652-1.642)$ \\
\hline Hyperlipidemia & 0.001 & $2.270(1.411-3.653)$ & $<0.001$ & 2.792 (1.772-4.399) & 0.001 & $2.257(1.403-3.631)$ & $<0.001$ & $2.548(1.616-4.015)$ & 0.001 & $2.272(1.410-3.659)$ & $<0.001$ & $2.806(1.781-4.422)$ \\
\hline \multicolumn{13}{|l|}{ Smoking duration } \\
\hline Group 1 (0) & \multicolumn{2}{|c|}{ Reference } & \multicolumn{2}{|c|}{ Reference } & \multicolumn{2}{|c|}{ Reference } & \multicolumn{2}{|c|}{ Reference } & & Reference & & \\
\hline Group 2 (>0, $\leq 15)$ & 0.087 & $2.158(0.895-5.203)$ & 0.991 & $1.005(0.422-2.393)$ & 0.098 & $2.106(0.872-5.084)$ & 0.860 & $1.081(0.454-2.576)$ & 0.092 & $2.131(0.884-5.140)$ & 0.979 & $1.012(0.426-2.404)$ \\
\hline Group 3 (>15, $\leq 30)$ & 0.324 & $0.715(0.367-1.393)$ & 0.101 & $0.596(0.321-1.106)$ & 0.326 & $0.716(0.367-1.396)$ & 0.112 & $0.604(0.325-1.125)$ & 0.340 & $0.723(0.371-1.408)$ & 0.128 & $0.619(0.334-1.147)$ \\
\hline Group 4 (>30) & 0.405 & $1.333(0.678-2.617)$ & 0.816 & $0.926(0.486-1.764)$ & 0.408 & $1.331(0.676-2.623)$ & 0.994 & $0.997(0.523-1.903)$ & 0.421 & $1.319(0.672-2.591)$ & 0.754 & $0.902(0.475-1.716)$ \\
\hline SBP, mmHg & 0.228 & $1.009(0.995-1.023)$ & 0.767 & $1.002(0.989-1.016)$ & 0.224 & 1.009 (0.995-1.023) & 0.925 & $1.001(0.987-1.014)$ & 0.204 & $1.009(0.995-1.024)$ & 0.733 & $1.002(0.989-1.016)$ \\
\hline $\mathrm{DBP}, \mathrm{mmHg}$ & 0.039 & 0.976 (0.954-0.999) & 0.014 & $0.973(0.952-0.994)$ & 0.044 & $0.977(0.955-0.999)$ & 0.017 & $0.974(0.953-0.995)$ & 0.031 & 0.975 (0.953-0.998) & 0.013 & $0.973(0.952-0.994)$ \\
\hline $\mathrm{UA}, \mu \mathrm{mol} / \mathrm{L}$ & 0.931 & $1.000(0.998-1.002)$ & 0.015 & 0.997 (0.995-0.999) & 0.785 & $1.000(0.997-1.002)$ & 0.010 & $0.997(0.995-0.999)$ & 0.931 & $1.000(0.998-1.002)$ & 0.020 & $0.997(0.995-1.000)$ \\
\hline $\mathrm{HCY}, \mu \mathrm{mol} / \mathrm{L}$ & 0.696 & $0.996(0.979-1.014)$ & 0.961 & $1.000(0.985-1.016)$ & 0.775 & $0.997(0.980-1.015)$ & 0.905 & $1.001(0.986-1.017)$ & 0.675 & $0.996(0.980-1.013)$ & 0.974 & $1.000(0.985-1.015)$ \\
\hline Tbil, $\mu \mathrm{mol} / \mathrm{L}$ & \multicolumn{2}{|c|}{ P-trend: 0.678} & \multicolumn{2}{|c|}{ P-trend: $<0.001$} & & & & & & & & \\
\hline Tertile1 (<8.6) & 0.737 & $1.108(0.611-2.009)$ & $<0.001$ & $3.502(2.018-6.075)$ & & & & & & & & \\
\hline Tertile2 (8.6-12.1) & 0.518 & $1.182(0.712-1.961)$ & 0.226 & $1.354(0.829-2.213)$ & & & & & & & & \\
\hline Tertile3 (>12.1) & \multicolumn{2}{|c|}{ Reference } & \multicolumn{2}{|c|}{ Reference } & & & & & & & & \\
\hline Dbil, $\mu \mathrm{mol} / \mathrm{L}$ & & & & & \multicolumn{2}{|c|}{ P-trend: 0.145} & \multicolumn{2}{|c|}{ P-trend: $<0.001$} & & & & \\
\hline Tertile $1(<3.7)$ & & & & & 0.161 & $1.544(0.841-2.835)$ & $<0.001$ & $4.199(2.380-7.410)$ & & & & \\
\hline Tertile $2(3.7-5.3)$ & & & & & 0.198 & $1.393(0.841-2.307)$ & 0.061 & $1.592(0.979-2.589)$ & & & & \\
\hline Tertile 3 (>5.3) & & & & & \multicolumn{2}{|c|}{ Reference } & \multicolumn{2}{|c|}{ Reference } & & & & \\
\hline lbil, $\mu \mathrm{mol} / \mathrm{L}$ & & & & & & & & & \multicolumn{2}{|c|}{ P-trend: 0.751} & \multicolumn{2}{|c|}{ P-trend: < 0.001} \\
\hline Tertile $1(<4.6)$ & & & & & & & & & 0.736 & $1.113(0.598-2.069)$ & $<0.001$ & $3.149(1.758-5.641)$ \\
\hline Tertile 2 (4.6-7.9) & & & & & & & & & 0.937 & $1.021(0.605-1.724)$ & 0.354 & $1.271(0.765-2.113)$ \\
\hline Tertile 3 (>7.9) & & & & & & & & & \multicolumn{2}{|c|}{ Reference } & \multicolumn{2}{|l|}{ Reference } \\
\hline
\end{tabular}

None-mild: stenosis 0-49\%, moderate: stenosis 50-69\%, severe-occlusion group: stenosis 70-100\%. ICAS, intracranial atherosclerotic stenosis; SBP, systolic blood pressure; DBP, diastolic blood pressure; UA, uric acid; HCY, homocysteine; Tbil, total bilirubin; Dbil, direct bilirubin; Ibil, indirect bilirubin. Smoking duration: group 1 (0, non-smoker), group 2 (0-15 pack-years), group 3 (15-30 pack-years) and group 4 (>30 pack-years). 
TABLE 5 | Multivariate logistic regression analyses for predictors of multi-stenosis of ICAS.

\begin{tabular}{|c|c|c|c|c|c|c|c|c|c|c|c|}
\hline \multicolumn{2}{|c|}{2 vs. 1} & \multicolumn{2}{|c|}{$\geq 3$ vs. 1} & \multicolumn{2}{|c|}{2 vs. 1} & \multicolumn{2}{|c|}{$\geq 3$ vs. 1} & \multicolumn{2}{|c|}{2 vs. 1} & \multicolumn{2}{|c|}{$\geq 3$ vs. 1} \\
\hline$P$-value & OR $(95 \% \mathrm{Cl})$ & $P$-value & OR (95\% Cl) & $P$-value & OR (95\% Cl) & $P$-value & OR (95\% Cl) & $P$-value & OR (95\% Cl) & $P$-value & OR $(95 \% \mathrm{Cl})$ \\
\hline
\end{tabular}

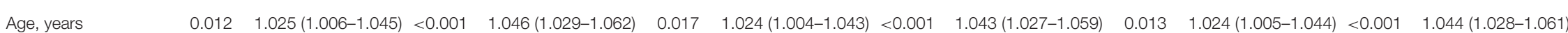

Sex, (Female vs. male)

$\begin{array}{llllllllllll} & 1.025(1.006-1.045) & <0.001 & 1.046(1.029-1.062) & 0.017 & 1.024(1.004-1.043) & <0.001 & 1.043(1.027-1.059) & 0.013 & 1.024(1.005-1.044) & <0.001 & 1.044(1.028-1.061)\end{array}$

Hypertension

$\begin{array}{lllllllll}0.943 & 0.980(0.565-1.700) & 0.663 & 0.905(0.577-1.419) & 0.875 & 0.957(0.553-1.656) & 0.564 & 0.878(0.564-1.366)\end{array}$

$\begin{array}{lllll}0.905 & 0.967(0.558-1.676) & 0.624 & 0.894(0.573-1.397)\end{array}$

Diabetes mellitus

$0.013 \quad 1.826(1.136-2.934) \quad 0.001 \quad 1.930(1.321-2.818)$

Hyperlipidemia

Group 1 (0)

Group $2(>0, \leq 15)$ $\begin{array}{llllllll}0.003 & 0.525(0.344-0.801) & 0.135 & 0.769(0.545-1.085) & 0.003 & 0.531(0.349-0.807) & 0.118 & 0.764(0.544-1.071)\end{array}$

$0.003 \quad 0.526(0.345-0.802)$

$0.6261 .118(0.714-1.750)$

Reference

Reference

Reference

Reference

Reference

$\begin{array}{llllllllllll}0.913 & 0.956(0.427-2.142) & 0.833 & 1.071(0.564-2.036) & 0.851 & 0.925(0.414-2.070) & 0.830 & 1.072(0.569-2.019) & 0.962 & 0.981(0.438-2.194) & 0.819 & 1.077(0.569-2.038)\end{array}$ $\begin{array}{lllllllllllll}0.739 & 1.124(0.566-2.231) & 0.421 & 1.259(0.719-2.204) & 0.693 & 1.147(0.579-2.272) & 0.357 & 1.296(0.746-2.253) & 0.669 & 1.161(0.586-2.299) & 0.324 & 1.322(0.759-2.302)\end{array}$ $\begin{array}{lllllllllllll}0.925 & 1.029(0.569-1861) & 0.104 & 0.660(0.399-1.089) & 0.977 & 1.009(0.560-1.818) & 0.099 & 0.661(0.404-1.081) & 0.886 & 1.044(0.578-1.888) & 0.087 & 0.648(0.394-1.066)\end{array}$ $\begin{array}{llllllllllllll}0.580 & 1.027(0.934-1.131) & 0.440 & 0.967(0.887-1.054) & 0.557 & 1.029(0.936-1.131) & 0.454 & 0.968(0.890-1.054) & 0.516 & 1.033(0.937-1.137) & 0.470 & 0.969(0.890-1.055)\end{array}$ $\begin{array}{lllllllllllll}0.121 & 1.174(0.959-1.436) & 0.014 & 1.244(1.045-1.481) & 0.105 & 1.181(0.966-1.444) & 0.012 & 1.247(1.050-1.482) & 0.124 & 1.173(0.957-1.437) & 0.015 & 1.240(1.042-1.475)\end{array}$

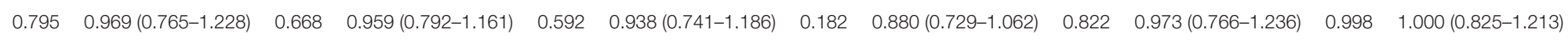
P-trend: $0.101 \quad P$-trend: $<0.001$

$0.1201 .484(0.902-2.441)<0.001 \quad 3.540(2.365-5.298)$ $0.003 \quad 2.052(1.270-3.314)<0.001 \quad 3.470(2.312-5.208)$

Group $4(>30)$

$\mathrm{FBG}, \mathrm{mmol}$

HbA1c, \%

LDL, $\mathrm{mm}$

Tbil, $\mu \mathrm{mol} / \mathrm{L}$

Tertile $1(<8.4)$

Tertile 2 (8.4-11.9)

Reference

Reference

Dbil, $\mu \mathrm{mol} / \mathrm{L}$

Tertile $1(<3.6)$

Tertile $2(3.6-5.1)$

Tertile $3(>5.1)$

Ibil, $\mu \mathrm{mol} / \mathrm{L}$

Tertile $1(<4.5)$

Tertile $2(4.5-6.9)$

Tertile $3(>6.9)$

P-trend: $0.610 \quad$ P-trend: $<0.001$

$0.617 \quad 1.136(0.689-1.874)<0.001 \quad 2.585(1.743-3.835)$

$0.0681 .552(0.967-2.491)<0.001 \quad 2.110(1.416-3.144)$

Reference

1, single-vessel stenosis; 2, two-vessel stenosis; $\geq 3$, multiple-vessel stenosis. ICAS, intracranial atherosclerotic stenosis; FBG, fasting blood glucose; HbA1c, glycosylated hemoglobin A1c; LDL, low density lipoprotein; Tbil, total bilirubin; Dbil, direct bilirubin; Ibil, indirect bilirubin. Smoking duration: group 1 (0, non-smoker), group 2 (0-15 pack-years), group 3 (15-30 pack-years) and group 4 (>30 pack-years).

P-trend: $0.136 \quad$ P-trend: $<0.001$

$0.1451 .461(0.877-2.434)<0.001 \quad 3.552(2.361-5.345)$ $0.009 \quad 1.866(1.167-2.986)<0.001 \quad 2.384(1.604-3.545)$ Reference Reference 
foam cell formation, leading to atherosclerosis and ultimately cerebral ischemia (37-39). Bilirubin, as the main end-product of heme metabolism, can scavenge free radicals and reduce the production of reactive oxygen species, thereby reducing the progression of atherosclerosis $(40,41)$. Second, a study by Vachharajani et al. showed that bilirubin could down-regulate the expression of $\mathrm{P}$ - and $\mathrm{E}$ - selectin induced by endotoxin, thus accounting for its anti-platelet aggregation effect (42). It has also been demonstrated that high concentrations of Ibil, as seen in Gilbert syndrome, could inhibit platelet aggregation induced by collagen and adenosine diphosphate (43). Third, bilirubin is negatively correlated with inflammatory markers, such as C-reactive protein, neutrophil-leukocyte ratio, and red cell distribution width, indicating that bilirubin could reduce pro-inflammatory cytokines and might also inhibit the inherent inflammatory process of atherosclerosis (44). Fourth, bilirubin could dissolve and transport cholesterol. Patients with hereditary diseases associated with elevated bilirubin levels have increased high-density lipoprotein/LDL ratio and decreased apolipoprotein B/apolipoprotein A-1 and total cholesterol levels (43). Fifth, previous research has established that bilirubin could delay the progression of atherosclerosis and improve vessel wall elasticity by down-regulating matrix metalloproteinase (45-47).

Accumulating clinical evidence proves that bilirubin has a protective impact on the carotid artery (48), cardiovascular system (9), and peripheral blood vessels (49). Nevertheless, there has been little discussion about the association between bilirubin and ICAS. In 2020, a population-based cross-sectional study pointed that serum Tbil, Dbil, and Ibil levels were negatively interrelated with aICAS, which is in accordance with our results (15). However, this paper focused on the connection between bilirubin (including Tbil, Dbil, and Ibil) and aICAS rather than sICAS. In addition, some scholars have examined the influence of serum Tbil on cerebral atherosclerosis and cerebral small vessel disease in the same subject and found that serum Tbil levels were negatively correlated with cerebral atherosclerosis (16). A study by Chen et al. revealed that Ibil concentrations increase with the exacerbation of intracranial or extracranial atherosclerotic stenosis, but decrease in patients with cranial vascular occlusion. In addition, there was no correlation between serum Tbil and Dbil levels and ICAS in their study (50). This is inconsistent with our results. A possible explanation for this might be that only 189 patients were recruited and they were divided into normal, mild $(<50 \%)$, moderate $(50-$ $69 \%)$, severe (70-99\%) and occlusion groups in their research. Meanwhile, the patients' gender, hypertension, smoking and alcohol consumption histories in each group were not completely matched in their study.

Hyperlipidemia is a well-established risk factor of macroangiopathy. However, our results showed that the presence of hyperlipidemia is a negative predictor for multistenosis of ICAS in the regression model of Table 5. One possible explanation for this paradox could be that the variable "hyperlipidemia" (yes/no) is rather imprecise when accounting a patient's risk for atherosclerosis. In our study, hyperlipidemia cases also included patients who had a history of hyperlipidemia or were currently receiving anti-hyperlipidemia therapy, while their present blood lipid levels may be within normal ranges. This was also supported by the unremarkable differences in LDL concentrations among the three groups (single-, two- and multiple-vessel stenosis) in patients with diagnosis of hyperlipidemia.

In this study, the bilirubin levels were significantly lower in patients with severe-occlusion or multiple-vessel stenosis, however, no clear linear dose-effect relationship between bilirubin levels and the extent of ICAS could be extrapolated from our data. The potential reasons are that the sample size of the none-mild group is relatively small and MRA was used to assess the degree of ICAS in the majority of patients, which is not the gold standard. MRA may amplify the extent of ICAS due to vascular tortuosity and various artifacts, thus causing diagnostic bias. Further research on the dose-effect-relationship between bilirubin levels and ICAS is needed.

There were some limitations in this study. First, it is a hospitalbased, descriptive, retrospective cross-sectional study, and the results are unable to demonstrate a causal relationship between sICAS and serum bilirubin levels. Second, the participants were recruited from a single-center, so one should be cautious in inferring the results to other populations. Third, we only recorded the baseline levels of serum bilirubin, which might have a dynamic change during the development of ischemic stroke. Fourth, the evaluation of sICAS was based on different imaging methods, which might lead to diagnostic bias. Further studies, preferably with multicenter design, are needed to be conducted to confirm our findings.

\section{CONCLUSION}

In conclusion, we found that lower bilirubin levels might indicate severe and multiple atherosclerotic stenoses of patients with sICAS in a Chinese Han population.

\section{DATA AVAILABILITY STATEMENT}

The raw data supporting the conclusions of this article will be made available by the authors, without undue reservation.

\section{ETHICS STATEMENT}

The studies involving human participants were reviewed and approved by Xiangya Hospital, Central South University. The patients/participants provided their written informed consent to participate in this study.

\section{AUTHOR CONTRIBUTIONS}

FY and LZ: methodology and writing-original draft preparation. ZL, YL, DL, and XF: investigation and data curation. JX: conceptualization and writing-reviewing and editing. All authors contributed to the article and approved the submitted version. 


\section{FUNDING}

This study was supported by the National Natural Science Foundation of China (Grant No. 81671166), the Fundamental Research Funds for the Central Universities of Central South University (Nos. 2019zzts902 and 2021zzts1033), the Provincial Key Plan for Research and Development of Hunan (Grant No. 2020SK2067), and the Project Program of National Clinical Research Center for Geriatric Disorders (Xiangya Hospital, Grant No. 2020LNJJ16).

\section{REFERENCES}

1. Wang Y, Zhao X, Liu L, Soo YO, Pu Y, Pan Y, et al. Prevalence and outcomes of symptomatic intracranial large artery stenoses and occlusions in China: the Chinese Intracranial Atherosclerosis (CICAS) Study. Stroke. (2014) 45:663-9. doi: 10.1161/STROKEAHA.113.003508

2. Wong KS, Li H, Chan YL, Ahuja A, Lam WW, Wong A, et al. Use of transcranial Doppler ultrasound to predict outcome in patients with intracranial large-artery occlusive disease. Stroke. (2000) 31:2641-7. doi: 10.1161/01.STR.31.11.2641

3. Man BL, Fu YP. Concurrent stenoses: a common etiology of stroke in Asians. World J Clin Cases. (2014) 2:201-5. doi: 10.12998/wjcc.v2.i6.201

4. Suwanwela NC, Chutinetr A. Risk factors for atherosclerosis of cervicocerebral arteries: intracranial versus extracranial. Neuroepidemiology. (2003) 22:37-40. doi: 10.1159/000067112

5. Shapiro SM. Definition of the clinical spectrum of kernicterus and bilirubin-induced neurologic dysfunction (BIND). J Perinatol. (2005) 25:54-9. doi: $10.1038 /$ sj.jp.7211157

6. Johnson L, Bhutani VK. The clinical syndrome of bilirubininduced neurologic dysfunction. Semin Perinatol. (2011) 35:101-13. doi: 10.1053/j.semperi.2011.02.003

7. Vítek L, Ostrow JD. Bilirubin chemistry and metabolism; harmful and protective aspects. Curr Pharm Des. (2009) 15:2869-83. doi: 10.2174/138161209789058237

8. Kang SJ, Lee C, Kruzliak P. Effects of serum bilirubin on atherosclerotic processes. Ann Med. (2014) 46:138-47. doi: 10.3109/07853890.2014.895588

9. Schwertner HA, Jackson WG, Tolan G. Association of low serum concentration of bilirubin with increased risk of coronary artery disease. Clin Chem. (1994) 40:18-23. doi: 10.1093/clinchem/40.1.18

10. Breimer LH, Wannamethee G, Ebrahim S, Shaper AG. Serum bilirubin and risk of ischemic heart disease in middle-aged British men. Clin Chem. (1995) 41:1504-8. doi: 10.1093/clinchem/41.10.1504

11. Zhang MM, Gao Y, Zheng YY, Chen Y, Liu F, Ma YT, et al. Association of fasting serum bilirubin levels with clinical outcomes after percutaneous coronary intervention: a prospective study. Cardiovasc Toxicol. (2017) 17:471-7. doi: 10.1007/s12012-017-9405-3

12. Luo Y, Li JW, Lu ZJ, Wang C, Guan DN, Xu Y. Serum bilirubin after acute ischemic stroke is associated with stroke severity. Curr Neurovasc Res. (2012) 9:128-32. doi: 10.2174/156720212800410876

13. Choi Y, Lee SJ, Spiller W, Jung KJ, Lee JY, Kimm H, et al. Causal associations between serum bilirubin levels and decreased stroke risk: a two-sample Mendelian randomization study. Arterioscler Thromb Vasc Biol. (2020) 40:437-45. doi: 10.1161/ATVBAHA.119.313055

14. Li RY, Cao ZG, Zhang JR, Li Y, Wang RT. Decreased serum bilirubin is associated with silent cerebral infarction. Arterioscler Thromb Vasc Biol. (2014) 34:946-51. doi: 10.1161/ATVBAHA.113.303003

15. Zhong K, Wang X, Ma X, Ji X, Sang S, Shao S, et al. Association between serum bilirubin and asymptomatic intracranial atherosclerosis: results from a population-based study. Neurol Sci. (2020) 41:1531-8. doi: 10.1007/s10072-020-04268-x

16. Kim J, Yoon SJ, Woo MH, Kim SH, Kim NK, Kim J, et al. Differential impact of serum total bilirubin level on cerebral atherosclerosis and cerebral small vessel disease. PLoS ONE. (2017) 12:e0173736. doi: 10.1371/journal.pone.0173736

\section{ACKNOWLEDGMENTS}

We thank all patients for their participation in this study. We also thank Dr. Yuanlin Ying for her help in language polishing.

\section{SUPPLEMENTARY MATERIAL}

The Supplementary Material for this article can be found online at: https://www.frontiersin.org/articles/10.3389/fneur. 2021.714098/full\#supplementary-material

17. Wang G, Fang B, Yu X, Li Z. Interpretation of 2018 guidelines for the early management of patients with acute ischemic stroke. Zhonghua Wei Zhong Bing Ji Jiu Yi Xue. (2018) 30:289-95. doi: 10.3760/cma.j.issn.2095-4352.2018.04.001

18. Gao S, Wang YJ, Xu AD, Li YS, Wang DZ. Chinese ischemic stroke subclassification. Front Neurol. (2011) 2:6. doi: 10.3389/fneur.2011.00006

19. Barnett HJ, Barnes RW, Clagett GP, Ferguson GG, Robertson JT, Walker PM. Symptomatic carotid artery stenosis: a solvable problem. North Am Symptomatic Carotid Endarterectomy Trial Stroke. (1992) 23:1048-53. doi: 10.1161/01.STR.23.8.1048

20. Zhou D, Ding J, Ya J, Pan L, Bai C, Guan J, et al. Efficacy of remote ischemic conditioning on improving WMHs and cognition in very elderly patients with intracranial atherosclerotic stenosis. Aging. (2019) 11:634-48. doi: 10.18632/aging.101764

21. Kimm H, Yun JE, Jo J, Jee SH. Low serum bilirubin level as an independent predictor of stroke incidence: a prospective study in Korean men and women. Stroke. (2009) 40:3422-7. doi: 10.1161/STROKEAHA.109.560649

22. Verdecchia P, Reboldi G, Angeli F. The 2020 International Society of Hypertension global hypertension practice guidelines - key messages and clinical considerations. Eur J Intern Med. (2020) 82:1-6. doi: 10.1016/j.ejim.2020.09.001

23. Basri NI, Mahdy ZA, Ahmad S, Abdul Karim AK, Shan LP, Abdul Manaf MR, et al. The World Health Organization (WHO) versus The International Association of Diabetes and Pregnancy Study Group (IADPSG) diagnostic criteria of gestational diabetes mellitus (GDM) and their associated maternal and neonatal outcomes. Horm Mol Biol Clin Investig. (2018) 34:20170077. doi: 10.1515/hmbci-2017-0077

24. Teramoto T, Sasaki J, Ishibashi S, Birou S, Daida H, Dohi S, et al. Diagnostic criteria for dyslipidemia. J Atheroscler Thromb. (2013) 20:655-60. doi: $10.5551 /$ jat.17152

25. Lee SM, Oh CM, Kim MH, Ha E, Hong M, Ryoo JH. Current smoking status as a predictor of cerebral infarction in men: a retrospective cohort study in South Korea. BMJ Open. (2021) 11:e042317. doi: 10.1136/bmjopen-2020-042317

26. Feng X, Yu F, Zhou X, Liu Z, Liao D, Huang Q, et al. MMP9 rs17576 is simultaneously correlated with symptomatic intracranial atherosclerotic stenosis and white matter hyperintensities in Chinese population. Cerebrovasc Dis. (2021) 50:4-11. doi: 10.1159/000511582

27. Ryu WS, Park SS, Kim YS, Lee SH, Kang K, Kim C, et al. Long-term natural history of intracranial arterial stenosis: an MRA follow-up study. Cerebrovasc Dis. (2014) 38:290-6. doi: 10.1159/000367587

28. Samuels OB, Joseph GJ, Lynn MJ, Smith HA, Chimowitz MI. A standardized method for measuring intracranial arterial stenosis. AJNR Am J Neuroradiol. (2000) 21:643-6.

29. Chung JW, Oh MJ, Cho YH, Moon GJ, Kim GM, Chung CS, et al. Distinct roles of endothelial dysfunction and inflammation in intracranial atherosclerotic stroke. Euro Neurol. (2017) 77:211-9. doi: 10.1159/000460816

30. Yu F, Lu J, Li Z, Zhou X, Zeng S, Zhan Q, et al. Correlation of plasma vascular endothelial growth factor and endostatin levels with symptomatic intra- and extracranial atherosclerotic stenosis in a Chinese Han population. J Stroke Cerebrovasc Dis. (2017) 26:1061-70. doi: 10.1016/j.jstrokecerebrovasdis.2016.12.021

31. Shimizu K, Shimomura K, Tokuyama Y, Sakurai K, Isahaya K, Takaishi $\mathrm{S}$, et al. Association between inflammatory biomarkers and progression of 
intracranial large artery stenosis after ischemic stroke. J Stroke Cerebrovasc Dis. (2013) 22:211-7. doi: 10.1016/j.jstrokecerebrovasdis.2011.07.019

32. Arenillas JF, Alvarez-Sabín J, Molina CA, Chacón P, FernándezCadenas I, Ribó $M$, et al. Progression of symptomatic intracranial large artery atherosclerosis is associated with a proinflammatory state and impaired fibrinolysis. Stroke. (2008) 39:1456-63. doi: 10.1161/STROKEAHA.107.498600

33. Forman HJ, Zhang H. Targeting oxidative stress in disease: promise and limitations of antioxidant therapy. Nat Rev Drug Discov. (2021). doi: 10.1038/s41573-021-00233-1. [Epub ahead of print].

34. Hansson GK. Atherosclerosis-an immune disease: the Anitschkov Lecture 2007. Atherosclerosis. (2009) 202:2-10. doi: 10.1016/j.atherosclerosis.2008.08.039

35. Stocker R, Yamamoto Y, McDonagh AF, Glazer AN, Ames BN. Bilirubin is an antioxidant of possible physiological importance. Science. (1987) 235:1043-6. doi: $10.1126 /$ science. 3029864

36. Jameel NM, Frey BM, Frey FJ, Gowda TV, Vishwanath BS. Inhibition of secretory phospholipase $\mathrm{A}(2)$ enzyme by bilirubin: a new role as endogenous anti-inflammatory molecule. Mol Cell Biochem. (2005) 276:21925. doi: 10.1007/s11010-005-4441-x

37. Kwiatkowska I, Hermanowicz JM, Mysliwiec M, Pawlak D. Oxidative storm induced by tryptophan metabolites: missing link between atherosclerosis and chronic kidney disease. Oxidative Med Cell Longevity. (2020) 2020:6656033. doi: $10.1155 / 2020 / 6656033$

38. Badran A, Nasser SA, Mesmar J, El-Yazbi AF, Bitto A, Fardoun MM, et al. Reactive oxygen species: modulators of phenotypic switch of vascular smooth muscle cells. Int J Mol Sci. (2020) 21:8764. doi: 10.3390/ijms21228764

39. Malekmohammad K, Sewell RDE, Rafieian-Kopaei M. Antioxidants and atherosclerosis: mechanistic aspects. Biomolecules. (2019) 9:301. doi: $10.3390 /$ biom 9080301

40. Stocker R, Glazer AN, Ames BN. Antioxidant activity of albuminbound bilirubin. Proc Natl Acad Sci U S A. (1987) 84:5918-22. doi: 10.1073/pnas.84.16.5918

41. Sedlak TW, Snyder SH. Bilirubin benefits: cellular protection by a biliverdin reductase antioxidant cycle. Pediatrics. (2004) 113:1776-82. doi: 10.1542/peds.113.6.1776

42. Vachharajani TJ, Work J, Issekutz AC, Granger DN. Heme oxygenase modulates selectin expression in different regional vascular beds. Am J Physiol Heart Circ Physiol. (2000) 278:H1613-7. doi: 10.1152/ajpheart.2000.278.5.H1613

43. Kundur AR, Singh I, Bulmer AC. Bilirubin, platelet activation and heart disease: a missing link to cardiovascular protection in Gilbert's syndrome? Atherosclerosis. (2015) 239:73-84. doi: 10.1016/j.atherosclerosis.2014.12.042

44. Akboga MK, Canpolat U, Sahinarslan A, Alsancak Y, Nurkoc S, Aras D, et al. Association of serum total bilirubin level with severity of coronary atherosclerosis is linked to systemic inflammation. Atherosclerosis. (2015) 240:110-4. doi: 10.1016/j.atherosclerosis.2015.02.051

45. Resveratrol inhibits human lung adenocarcinoma cell metastasis by suppressing heme oxygenase 1-mediated nuclear factor-kappaB pathway and subsequently downregulating expression of matrix metalloproteinases. Mol Nutr Food Res. (2010) 54(Suppl. 2):S196-204. doi: 10.1002/mnfr.200900550

46. Tanaka M, Fukui M, Tomiyasu KI, Akabame S, Nakano K, Hasegawa $\mathrm{G}$, et al. Low serum bilirubin concentration is associated with coronary artery calcification (CAC). Atherosclerosis. (2009) 206:287-91. doi: 10.1016/j.atherosclerosis.2009.02.010

47. Tanindi A, Erkan AF, Alhan A, Tore HF. Arterial stiffness and central arterial wave reflection are associated with serum uric acid, total bilirubin, and neutrophil-to-lymphocyte ratio in patients with coronary artery disease. Anatol J Cardiol. (2015) 15:396-403. doi: 10.5152/akd.2014.5447

48. Tao X, Wu J, Wang A, Xu C, Wang Z, Zhao X. Lower serum indirect bilirubin levels are inversely related to carotid intimamedia thickness progression. Curr Neurovasc Res. (2019) 16:148-55. doi: $10.2174 / 1567202616666190412153735$

49. Ozeki M, Morita H, Miyamura M, Fujisaka T, Fujita SI, Ito T, et al. High serum bilirubin is associated with lower prevalence of peripheral arterial disease among cardiac patients. Clin Chim Acta. (2018) 476:60-6. doi: 10.1016/j.cca.2017.11.013

50. Chen Y, Zhang X, Zhang L, Qin R, Xiao L. Protective and indicating effect of indirect bilirubin in intracranial or extracranial artery atherosclerotic stenosis progresses. Int J Clin Med. (2015) 6:512-9. doi: 10.4236/ijcm.2015. 67069

Conflict of Interest: The authors declare that the research was conducted in the absence of any commercial or financial relationships that could be construed as a potential conflict of interest.

Publisher's Note: All claims expressed in this article are solely those of the authors and do not necessarily represent those of their affiliated organizations, or those of the publisher, the editors and the reviewers. Any product that may be evaluated in this article, or claim that may be made by its manufacturer, is not guaranteed or endorsed by the publisher.

Copyright $\odot 2021$ Yu, Zhang, Liao, Luo, Feng, Liu and Xia. This is an open-access article distributed under the terms of the Creative Commons Attribution License (CC $B Y)$. The use, distribution or reproduction in other forums is permitted, provided the original author(s) and the copyright owner(s) are credited and that the original publication in this journal is cited, in accordance with accepted academic practice. No use, distribution or reproduction is permitted which does not comply with these terms. 